\begin{tabular}{|c|}
\hline Case \\
report \\
\hline
\end{tabular}

\title{
Gonococcal scalp abscess in a neonate delivered by caesarean section
}

\author{
E Varady, H Nsanze, T Slattery
}

Gonococcal infection in caesarean delivered babies is very rare and is usually limited to ophthalmia neonatorum. The mother had rupture of membranes 14 hours before the caesarean section. The infection was most likely introduced by the fetal scalp electrode probes applied 2 hours before delivery. This is the first reported case of a neonatal gonococcal abscess in a caesarean delivered infant.

(Sex Transm Inf 1998;74:451)

Keywords: gonococcal abscess; caesarean section

\section{Introduction}

Gonorrhoea is uncommon in the United Arab Emirates and very few cases of gonococcal ophthalmia neonatorum are seen. Gonorrhoea has been associated with premature delivery in South Africa, thus implicating the amniotic fluid. ${ }^{1}$ Gonococcal chorioamnionitis has been described in a patient with intact membranes. ${ }^{2}$ One case of gonococcal ophthalmia neonatorum in a preterm infant delivered by caesarean section, 22 hours after the rupture of membranes, has also been reported. ${ }^{3}$

\section{Case report}

A full term newborn delivered by an emergency caesarean section at 37-38 weeks' gestation, developed an abscess on the scalp. On the fourth day, when the baby was reviewed, there was a microabscess over the left parietal area measuring about $1 \mathrm{~cm}$ in diameter with a small crust on top and surrounded by a small erythematous ring.

The microabscess was punctured with a sterile needle and the pus drained. The baby was given two doses of intravenous vancomycin, followed by oral flucloxacillin for 3 days. The swelling resolved and the baby was discharged on oral flucloxacillin to be continued for further 3 days at home.

On microscopic examination, the specimen revealed many pus cells but no organisms. Culture grew Neisseria gonorrhoeae which was $\beta$ lactamase negative, and an initial direct sensitivity test (assuming a likely staphylococcal infection) showed sensitivity to co-amoxyclav, flucloxacillin, erythromycin, but resistance to vancomycin.

The indication for caesarean section was an unstable lie, fetal distress in the first stage of labour and failure to progress. Fetal scalp electrode application was attempted unsuccessfully at 4 hours and then successfully at 2 hours before the caesarean section. The baby was delivered via low segment caesarean section with an excellent Apgar score and weighed
2800 g. The mother had developed a low grade temperature on the first postpartum day.

In this pregnancy, the antenatal care had been adequate and routine serological tests for RPR, HIV, HbsAg, and rubella were negative. The father had low titres of Chlamydia trachomatis IgG but the mother was negative. Later, the parents were interviewed but were both symptom free and refused further investigations; however, they accepted treatment for gonorrhoea.

\section{Discussion}

The incidence of gonorrhoea is very low in the Arabian peninsula and gonococcal ophthalmia neonatorum is also expected to be extremely rare. ${ }^{4}$ However, a few adults in the region acquire sexually transmitted infections in various ways. This family had unrecognised gonorrhoea but no evidence of other sexually transmitted diseases. Refusal to complete the investigations for the family is a common problem in this population. Although gonorrhoea has been associated with premature delivery ${ }^{5}$ and there is increased bacterial conjunctival flora at delivery in babies delivered by caesarean after membrane rupture, apart from ophthalmia neonatorum, no other type of infections have been reported. In this report, the newborn developed an abscess after caesarean delivery. This is a rare occurrence and was considered to be the result of the electrode probe insertion.

1 Donders GG, Desmyter J, De Wet HD, V et al. The association of gonorrhoea and syphilis with premature birth and low birth weight. Genitourin Med 1993;69:98-101.

2 Smith LG Jr, Summers PR, Miles RW, et al. Gonococcal chorioamnionitis associated with sepsis: a case report. $A m \mathcal{F}$ Obstet Gynecol 1989;160:573-4.

3 Jacobsen T, Knudsen JD, Weis NM. Gonorrheal ophthalmia neonatorun in a premature infant delivered by caesarean section. Ugesk Laeger 1991;153:2571.

4 Nsanze H, Dawodu A, Usmani A, S et al. Ophthalmia neonatorum in the United Arab Emirates. Ann Trop Paediatr 1996;16:27-32.

5 Isenberg SJ, Apt L, Yoshimori R, et al. Source of the conjunctival bacterial flora at birth and implications for ophthalmia neonatorum prophylaxis. Am $\mathcal{F}$ Ophthalmol 1988;106:458-62. 